\title{
Régimen crítico en canales rectangulares de fondo curvo y sus aplicaciones
}

\author{
G. Sotelo-Ávila e I.I. Valdez-Izaguirre \\ D epartamento de Ingeniería Hidráulica \\ División de Ingeniería Civil, Topográfica y Geodésica. Facultad de Ingeniería, U N A M \\ E-mails: soteloa@servidor.unam.mx; ii_valdez_i@yahoo.com.mx
}

(recibido: mayo de 2001; aceptado: febrero de 2003)

\begin{abstract}
Resumen
El comportamiento del flujo en una curva vertical de un canal es diferente del rectilíneo, dado que la curvatura apreciable de las líneas de corriente modifica la distribución de la presión y velocidad en cada sección por el efecto que impone la fuerza centrífuga. En este artículo se presenta un desarrollo alternativo para obtener las condiciones del estado crítico del flujo curvilíneo permanente en canales rectangulares, tomando como hipótesis la ley del vórtice libre para la distribución de la velocidad en la sección. El cumplimiento de las ecuaciones del régimen crítico permite ubicar la sección de control y a partir de ésta, calcular el perfil del flujo en curvas verticales.
\end{abstract}

Descriptores: flujo en curvas verticales, régimen crítico en flujo curvilíneo, sección de control en flujo curvilíneo.

\begin{abstract}
The flow behaviour in a ver ti cally curved bot tom chan nel is differ ent from theoneon a flat bot tom chan nel. Thecen trifu gal forceaf fects thepres sureand velocity dis tri bu tionsand the stream lines are curved following bot tom thechan nel trend.

With thefreevor tex law as hy poth eses, theequa tionsfor thecriti cal con di tions in a rect an glt lar chan nel with curvilinear flow areob tained as shown. Knowing the criti cal con di tionsit will al lowlocating thecon trol section and thereforecal cu lating theflow profilebeforean daf ter the curve.
\end{abstract}

Keywords: flow in ver ti cally curved, crit i cal con di tions in curvilinear flow, con trol section in curvilinear flow.

\section{Aspectos generales}

El perfil del fondo de un canal generalmente se diseña ajustándolo a las condiciones topográficas y geológicas del sitio, mediante tramos rectos de fondo plano y diferentes pendientes unidos por curvas verticales, normalmente circulares o parabólicas.

Los cambios del fondo en dirección ver tical también se requieren para unir dos tramos de pendiente distinta, o bien, cuando es necesario proporcionar una 
determinada geometría al perfil del fondo por razones hidráulicas. Esto último puede presentarse al diseñar un cimacio y la conexión entre el pie de un cimacio y su canal de descarga, o bien, al diseñar una cubeta deflectora.

Cuando el canal tiene deflexiones en la dirección vertical y las líneas de corriente tienen curvatura apreciable, se modifica la distribución de la presión en cada sección por el efecto que impone la aceleración centrífuga. Las curvas verticales pueden ser cóncavas (figura 1a) o convexas (figura 1b). La curvatura pro duce un componente de la aceleración perpendicular a la dirección del flujo en ambas, el cual desvía la presión de la ley hidrostática, representada por la línea $A C$ cuando el flujo es recto, a la línea $A C^{\prime}$ cuando el flujo es curvilíneo. La fuerza centrífuga actúa hacia el ex te rior de la curva en ambos casos, pero en el flujo cóncavo pro duce una presión mayor que la hidrostática, mientras que en el convexo pro duce una menor.

El tratamiento adecuado del flujo curvilíneo es considerarlo como irrotacional y analizarlo mediante una red de flujo o con soluciones analíticas, como lo hizo Lenau y Cassidy (1969); Prasad (1984); sin em bargo, en ambos casos, el procedimiento resulta muy elaborado y a veces tedioso. Por lo anterior, se presenta un planteamiento diferente basado en que la distribución de la velocidad sigue la ley del vórtice libre con el fin de determinar las condiciones en que se realiza el flujo curvilíneo unidimensional en un canal rectangular con curvatura vertical. Con estos desarrollos se proponen las ecuaciones necesarias para analizar las condiciones del régimen crítico en un canal rectangular de fondo curvo, cuya aplicación proporciona resultados suficientemente precisos para fines prácticos.

\section{Flujo curvilíneo como un vórtice libre}

El flujo irrotacional tiene la propiedad de que la energía permanece constante en cualquier punto de una misma línea de corriente. Cuando el flujo es además curvo, a dicha propiedad se agrega que en cada punto de dicha línea se satisface la ecuación

$$
\frac{d v}{v}=-\frac{d r}{r}
$$

donde $r$ y $v$ son el radio local de curvatura de la línea de corriente y la velocidad local, respectivamente (ambos en el punto en cuestión). Se considera que las líneas de corriente en la sección del flujo cóncavo (figura 1a) asume la forma circular del fondo con un solo centro de curvatura, no obstante que se ven influenciadas desde un punto antes del cambio de dirección y en un tramo después del mismo; es decir, existe una zona de transición antes y después de la curva donde también se producen cambios en la distribución de la presión. La hipótesis permite integrar la ecuación 1 y obtener $\ln (v r)=$ constante, 0 bien, $v r=k$, que es la ley del vórtice libre, donde $k$ es constante para todas las líneas de corriente en una misma sección de la curva. Por tanto, se satisface que $v r=v_{a} r_{a}=k$, donde $v_{a}$ y $r_{a}$ son la velocidad y el radio de curvatura en un punto sobre la línea de corriente que coincide con la superficie libre de la misma sección.

Con una distribución de la velocidad siguiendo la ley del vórtice libre, $v=v_{a} r_{a} / r_{a}$, la velocidad media en una sección rectangular ortogonal al fondo es

$V=\frac{1}{d} \int_{r_{a}}^{R} v d r=\frac{r_{a} v_{a}}{\mathrm{~d}} \int_{r_{a}}^{R} \frac{d r}{r}=\frac{r_{a}}{d} \ln \left(\frac{R}{r_{a}}\right) v_{a}$ 


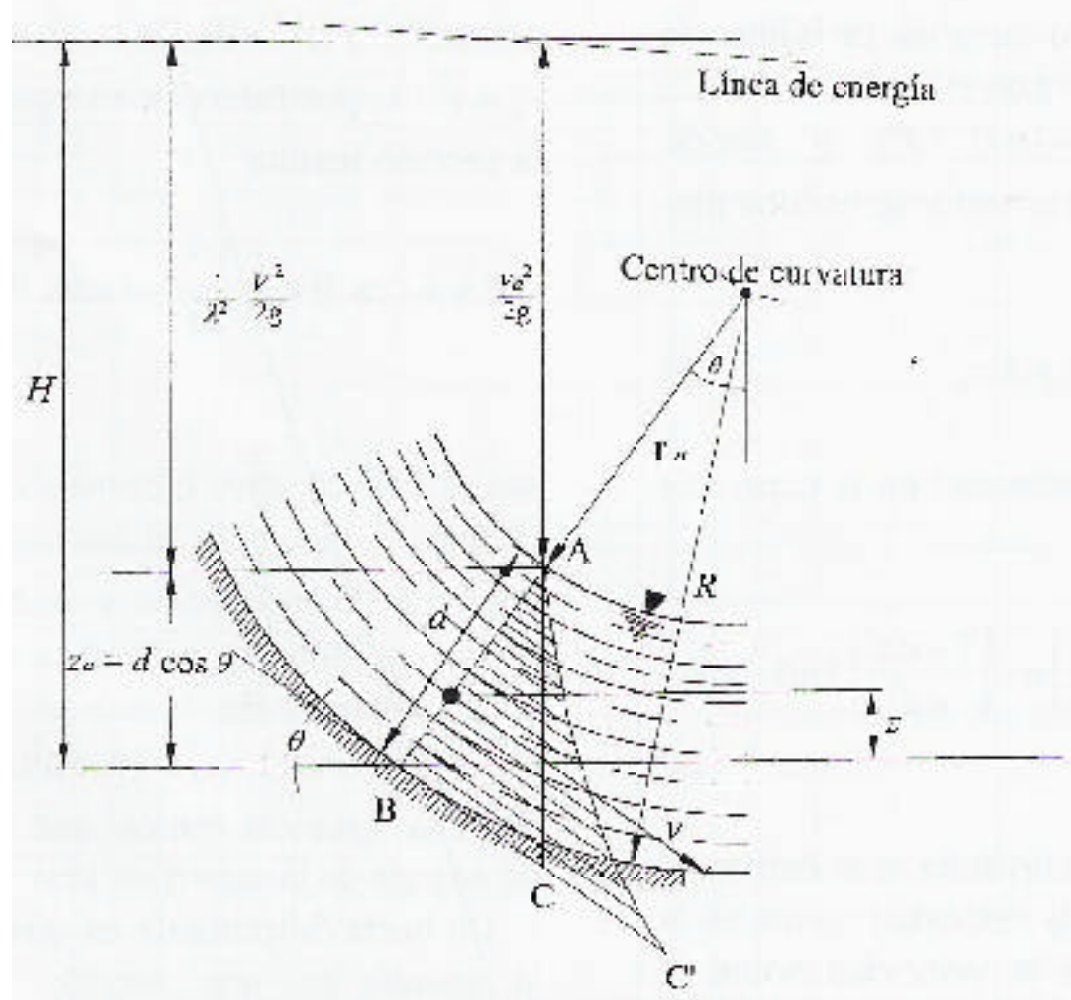

a) Flujo cóncavo

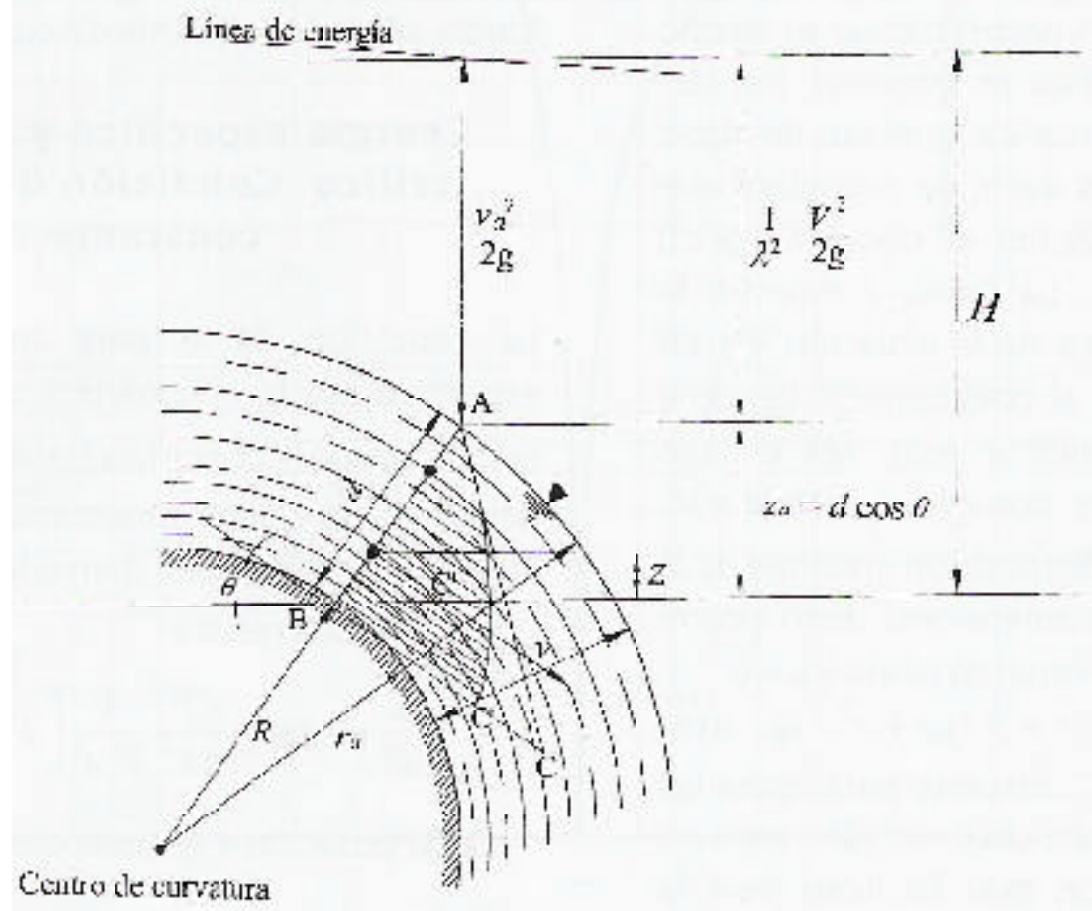

b) Llujo convexo

Figura 1. Distribución de la presión en el flujo curvilíneo 
donde $R$ es el radio de curvatura de la línea de corriente que co in cide con el fondo.

Pero $\ln \left(R / r_{a}\right)=-\ln \left(r_{a} / R\right)$, y siendo $r_{a}=R-d$, la ecuación anterior se escribe me- jor en la forma

$$
V=\lambda v_{a}
$$

donde $v_{a}$ es la velocidad en la superficie libre y

$$
\lambda=\left[1-\frac{R}{d}\right] \ln \left[1-\frac{d}{R}\right]=-\left[\frac{1-\kappa d}{\kappa d}\right] \ln [1-\kappa \mathrm{d}]
$$

es el coeficiente de curvatura que corrige el efecto de considerar la velocidad media en la sección, en lugar de la velocidad sobre la superficie libre, siendo el término $\kappa=1 / R$ la curvatura del fondo del canal y $d$ el tirante medido en dirección perpendicular al mismo fondo. Cuando el flujo es convexo, los términos que contienen a $\kappa d$ cambian de signo en la ecuación 4 , es decir, se considera que $\kappa>0$ cuando la curvatura es cóncava y $\kappa<0$ cuando es convexa. La figura 2 muestra la representación gráfica de la ecuación 4 y en ella se observa que el coeficiente $\lambda$ tiende a uno cuando $\kappa d$ tiende a cero, sea el flujo cóncavo o convexo y, con ello, $v_{a}$ tiende a $V$, es decir, tiende a la distribución uniforme de la velocidad media unidimensional. Esto ocurre cuando el fondo del canal es plano y $\kappa=0$.

La energía $H=z+p / g \rho+v^{2} / 2 g$ (respecto del fondo) es constante para todas las líneas de corriente en una sección, pero su valor se obtiene con más facilidad para la línea que coincide con la superficie libre del agua $(z=d \cos \theta)$, donde la presión es la atmosférica $\left(p_{a}=0\right)$. De la ecuación 3 resulta
$v_{a}=V / \lambda$; por tanto, la energía específica en la sección resulta

$$
E=d \cos \theta+\frac{1}{\lambda^{2}} \frac{V^{2}}{2 g}=d \cos \theta+\frac{1}{\lambda^{2}} \frac{Q^{2}}{2 g b^{2} d^{2}}
$$

sea el flujo cóncavo o convexo. Para un gasto $Q$ dado y conocidos el ancho b del canal, el ángulo $\theta$ de inclinación y la curvatura $\kappa$ del fondo, la energía específica $E$ es función exclusivamente del tirante $d$. Toda vez que $\left(1 / \lambda^{2}\right)\left(V^{2} / 2 g\right)=v_{a}^{2} / 2 g$, este término repre-

senta la distancia vertical que separa la línea de energía de la superficie libre en el canal.

Un hecho importante es que, para calcular la energía en una sección del flujo con curvatura vertical, no es necesario conocer la distribución de la presión ni la que hay en el fondo, sólo el coeficiente de curvatura.

\section{Energía específica y régimen crítico. Condición de gasto constante}

La condición límite para que la energía específica sea la mínima $\left(E_{c}\right)$ con que puede fluir un gasto $Q$ conocido a través de la sección del canal, se puede determinar utilizando el criterio de la primera derivada $(d E / d d=0)$. Dicha derivada resulta

$$
\frac{d E}{d d}=\cos \theta-\frac{Q^{2}}{g b^{2}} \frac{1}{\lambda^{3} d^{3}}\left[d \frac{d \lambda}{d d}+\lambda\right]
$$

De la ecuación 4 se tiene que

$$
\frac{d \lambda}{d d}=\frac{\ln (1-\kappa d)}{\kappa d^{2}}+\frac{1}{d}
$$

Con la misma ecuación 4, la anterior se transforma en 


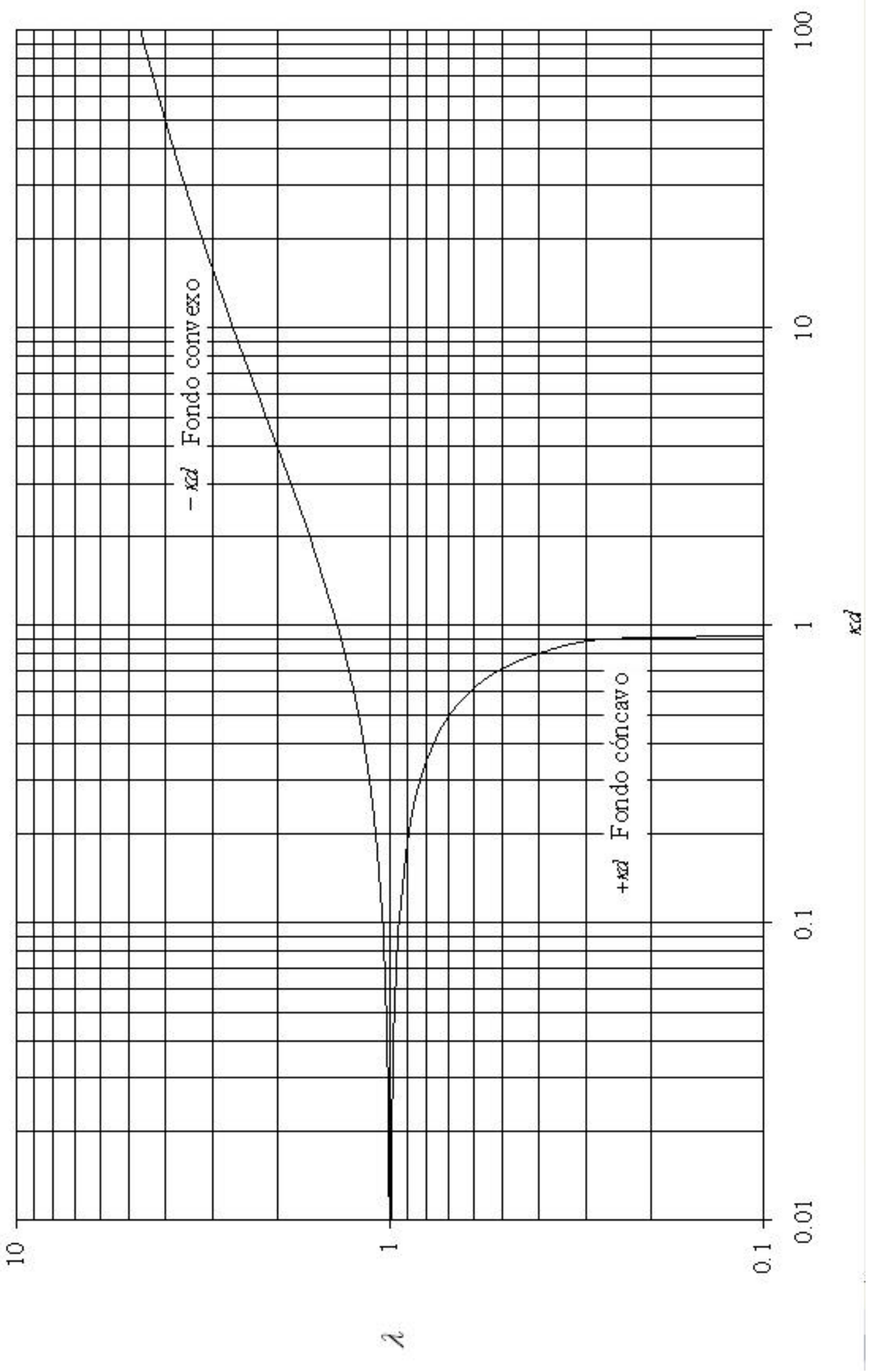


(8)

$$
\frac{d \lambda}{d d}=\frac{1}{d}\left(1-\frac{\lambda}{1-\kappa d}\right)
$$

Al sustituir la ecuación 8 en la 6 considerando el gasto unitario $q=\frac{Q}{b}$, se obtiene

$$
\frac{d E}{d d}=\cos \theta-\frac{q^{2}}{g \lambda^{3} d^{3}}\left[\frac{1-\kappa d(1+\lambda)}{1-\kappa d}\right]
$$

(9)

Para que $E$ sea mínima, debe ser $d E / d d=0$, por lo que la ecuación an te rior se convierte en

(10a)

$$
\frac{q^{2}}{g \cos \theta}=\left[\frac{\lambda^{3} d^{3}(1-\kappa d)}{1-\kappa d(1+\lambda)}\right]_{c}
$$

o bien

$$
\frac{q \kappa^{3 / 2}}{\sqrt{g \cos \theta}}=\left[-\frac{\lambda^{3}(\kappa d)^{3}(1-\kappa d)}{\kappa d(1+\lambda)-1}\right]_{c}^{1 / 2}
$$

expresión que representa la condición del estado crítico de un flujo curvilíneo en un canal rectangular. En ella se observa que el tirante crítico $d_{c}$ depende de la curvatura del fondo. La figura 3 muestra la representación gráfica de la ecuación $10 \mathrm{~b}$.

Con $q=V_{c} d_{c}$ en la ecuación 10a, resulta

(11)

$$
\frac{1}{\lambda^{2}} \frac{V_{c}^{2}}{2 g}=\frac{1}{2} \frac{d_{c} \cos \theta \lambda\left(1-\kappa d_{c}\right)}{1-\kappa d_{c}(1+\lambda)}
$$

siendo la energía específica mínima

$$
E_{c}=d_{c} \cos \theta\left[1+\frac{1}{2} \frac{\lambda\left(1-\kappa d_{c}\right)}{1-\kappa d_{c}(1+\lambda)}\right]
$$

Por otra parte, si se representa con $v_{0}$ a la velocidad de las partículas en el fondo de la sección, de la ecuación del vórtice libre: $v_{a} r_{a}=v_{0} R$, de manera que

$$
v_{0}=v_{a} \frac{R-d}{R}=v_{a}(1-\kappa d)
$$

donde, $v_{a}=V / \lambda=q / \lambda d$ de la ecuación 3; por tanto

$$
v_{0}=q\left(\frac{1-\kappa d}{\lambda d}\right)
$$

Un parámetro aplicable al flujo curvilíneo, relacionado con el número de Froude, de la ecuación 13 es

$$
\mathrm{F}=\frac{v_{0}}{\sqrt{g d \cos \theta}}=\frac{q}{\sqrt{g \cos \theta}} \frac{1-\kappa d}{\lambda d^{3 / 2}}
$$

el cual, para las condiciones críticas adquiere el valor

$$
\mathrm{F}_{\mathrm{c}}=\frac{q}{\sqrt{g \cos \theta}}\left[\frac{1-\kappa d}{\lambda d^{3 / 2}}\right]_{c}
$$

Pero, de la ecuación $10 \mathrm{~b}$ se tiene que

$$
\frac{q}{\sqrt{g \cos \theta}}=\left[-\frac{\lambda^{3} d^{3}(1-\kappa d)}{\kappa d(1+\lambda)-1}\right]_{c}^{1 / 2}
$$

Por tanto 


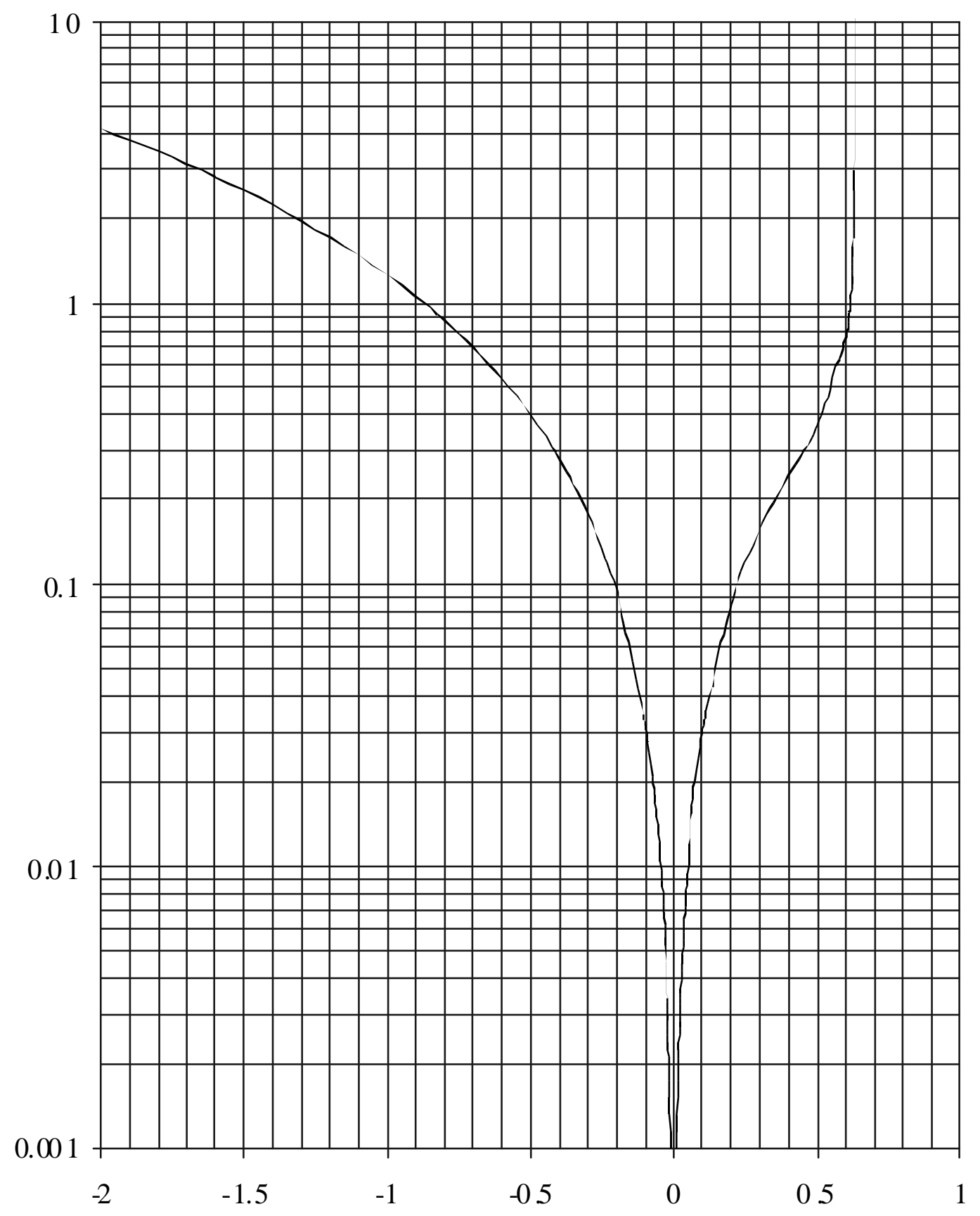




$$
\mathrm{F}_{\mathrm{c}}=\left[-\frac{\lambda(1-\kappa d)^{3}}{\kappa d(1+\lambda)-1}\right]_{c}^{1 / 2}
$$

El valor de $F_{c}$ no tiene que ser igual a uno ya que depende de $\lambda$ y de $\kappa d$. El número de Froude para cualquier condición del flujo curvilíneo se de fine ahora como

$$
F=\frac{F}{F}
$$

donde $F$ y $F_{c}$ están dados por las ecuaciones 14 y 17. Este número de Froude sí alcanza obviamente el valor $\mathrm{F}=1$ para el flujo crítico curvilíneo.

Cuando el fondo es plano $\kappa d_{c}=0, \lambda=1$ (Figura 2) y la ecuación 10a se transforma en $d_{c}=\sqrt[3]{q^{2} / g \cos \theta}$, que corresponde a la ecuación del tirante crítico para el flujo rectilíneo en canales rectangulares con fondo plano.

Para la misma condición de fondo plano, $\lambda=1$ y $\kappa d_{c}=0$, por tanto, de la ecuación 12 resulta que: $E_{c}=(3 / 2) d_{c} \cos \theta$ es la energía específica crítica del flujo rectilíneo en canales rectangulares.

Finalmente, de la ecuación 17 resulta $\mathrm{F}_{c}=1$ $y$, por tanto, para el fondo plano se obtiene la expresión convencional: $\mathrm{F}=V / \sqrt{g d \cos \theta}$, que coincide con el número de Froude convencional del flujo rectilíneo, de valor igual a 1 en condiciones críticas.

\section{Aplicaciones}

Curva de transición entre dos canales de pendiente distinta
La modificación de la presión en una curva vertical es más importante a medida que aumenta la velocidad y disminuye el radio de curvatura. El efecto se deja sentir tanto en flujo subcrítico como en supercrítico pero, en el último, la velocidad es mayor y los cambios de dirección ver ti cal se realizan con deflexiones más grandes y más frecuentes. Las curvas verticales bruscas deben evitarse a fin de prevenir perturbaciones del flujo en el canal. Las curvas verticales son más sencillas de considerar que las producidas en un cambio de dirección horizontal, pero el diseño de las curvas de conexión debe tomar en cuenta los siguientes aspectos:

a. Las curvas convexas deben ser suficientemente graduales con el fin de mantener presión positiva en el fondo y evitar que el flujo se separe. La presión negativa tiene que limitarse para evitar cavitación en los recubrimientos.

b. Las curvas cóncavas deben tener un radio de curvatura suficientemente grande con el fin de disminuir los efectos dinámicos sobre el fondo producidos por la fuerza centrífuga resultante del cambio de dirección.

c. La geometría de una curva vertical que une dos canales de pendiente menor a mayor debe ser lo más sencilla posible, preferentemente circular o parabólica con el fin de simplificar su construcción. En el caso de canales con flujo subcrítico de poca velocidad, los cambios de pendiente pueden ser bruscos si esto no implica erosión en el fondo.

Para evitar la tendencia del agua a separarse 


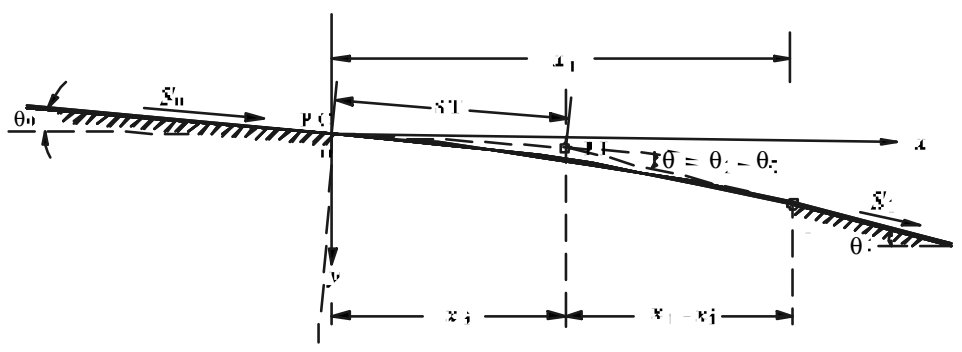

Figura 4. Curva vertical convexa

contacto, el perfil de una curva convexa debe ser considerablemente más tendido que la trayectoria del chorro libre lanzado con ángulo de inclinación $\theta_{0}$ y la energía específica $E_{0}$ del flujo en la sección donde inicia la curva. En la figura 4 se muestra la curva de conexión del fondo de dos ca na les de distinta pendiente.

Con el sistema coordenado mostrado, la forma de la curva queda definida por la ecuación de una parábola, como se demuestra en Sotelo (1994), que es

$$
y=x \tan \theta_{0}+\frac{x^{2}}{4 K E_{0} \cos ^{2} \theta_{0}}
$$

donde

$E_{0} \quad$ energía específica del flujo al iniciar la curvatura vertical de transición

$K$ factor que depende del grado de coincidencia deseado entre el perfil del fondo y del chorro descargado libremente

$\theta_{0} \quad$ ángulo de inclinación del fondo en el canal aguas arriba de la curva.

Para asegurar presión positiva sobre toda la superficie de contacto con el fondo, el valor de $K$ dehe cer inı ıl $\cap$ mavor de 15
La derivada de la ecuación 19 es

$$
\frac{d y}{d x}=\tan \theta_{0}+\frac{x}{2 K E_{0} \cos ^{2} \theta_{0}}
$$

Para obtener las coordenadas del punto en que la curva se vuelve tangente al fondo del segundo tramo, se iguala $d y / d x \operatorname{con} \tan \theta_{1}$ y resulta

$$
\tan \theta_{1}=\tan \theta_{0}+\frac{x_{1}}{2 K E_{0} \cos ^{2} \theta_{0}}
$$

Por tanto, la abscisa del punto 1 de tangencia es entonces

$$
x_{1}=2 K E \cos ^{2} \theta_{0}\left(\tan \theta_{1}-\tan \theta_{0}\right)
$$

y la ordenada $y_{1}$ se obtiene sustituyendo $x_{1}$ en la ecuación 19.

El perfil del fondo puede diseñarse con la forma parabólica dada por la ecuación 19 entre los puntos 0 y 1 . Para simplificar su geometría y construcción, el perfil puede cambiarse a una curva circular vertical que inicie en el origen 0 del sistema coordenado (punto PC) y termine en el punto 1 (punto PT), siendo $\mathrm{PI}$ el punto de inflexión y $\theta=\theta_{1}-\theta_{0}$ el ángulo de deflexión. 
La canalización de un río donde fluye un gasto de $15 \mathrm{~m}^{3} / \mathrm{s}$, se inicia con un tramo de $940 \mathrm{~m}$ de longitud, sección rectangular, ancho constante de $3 \mathrm{~m}$ y pendiente de 0.0042 también constante. Para descargar a otro río se dispone de una rápida que termine en una cubeta deflectora cuya geometría se muestra en la figura 5. Diseñar la curva de transición para el cambio de pendiente y determinar el tirante de la sección crítica considerando $n=0.015$ (Manning).

\section{Solución}

Debido a su gran longitud, se forma flujo uniforme en el primer tramo del canal, el cual debe ocurrir con tirante normal $y_{n}=1.4261 \mathrm{~m}$. En efecto, el área hidráulica es $A=42783 \mathrm{~m}^{2}$, el radio hidráulico $R_{h}=0.7311 \mathrm{~m}$, por tanto, la velocidad media del agua y el cau dal valen:

$$
\begin{gathered}
V=\frac{1}{0.015}(0.7311)^{2 / 3} \sqrt{0.0042}=3.5062 \mathrm{~m} / \mathrm{s} \\
Q=3.5062(4.2783)=15 \mathrm{~m}^{3} / \mathrm{s}
\end{gathered}
$$

El tirante crítico y la energía específica mínima en el primer tramo del canal (con fondo plano), para el gasto unitario $q=15 / 3=5 \mathrm{~m}^{2} / \mathrm{s}$ resultan los valores:

$$
\begin{gathered}
y_{c}=\left[\frac{(5)^{2}}{9.81}\right]^{1 / 3}=13659 \mathrm{~m} \\
E_{c}=\frac{3}{2}(13659)=2.04887 \mathrm{~m}
\end{gathered}
$$

En virtud de que $y_{n}>y_{c}$, el régimen es subcrítico en el primer tramo. Por efecto del curva vertical de transición entre las dos pendientes, por lo que dicha curva se diseña con la energía específica crítica antes calculada. El ángulo de inclinación del fondo del canal antes de la curva es $\theta_{0}=\tan ^{-1} 0.0042=0.24064^{\circ}$ y después de ella, $\theta_{1}=\tan ^{-1} 0.7=34.99202^{\circ}$.

Con $K=15$, de la ecuación 20 resulta

$$
\begin{gathered}
x_{1}=2(1.5)(2.0489)\left(\cos ^{2} 024064\right)(0.7-0.0042)= \\
42768 \mathrm{~m}
\end{gathered}
$$

De la ecuación 19:

$$
\begin{gathered}
y_{1}=4.2768(0.0042)+ \\
\frac{(42768)^{2}}{4(15)(2.0489) \cos ^{2} 0.24064^{\circ}}=15059 \mathrm{~m}
\end{gathered}
$$

El ángulo de deflexión total es

$$
\theta=\theta_{1}-\theta_{0}=34.99202-0.24064=34.75138^{\circ}
$$

La abscisa $x_{i}$ del punto PI de inflexión, se obtiene de cualquiera de las siguientes expresiones:

o bien

$$
x_{i}=\mathrm{ST} \cos \theta_{0},
$$

$$
x_{1}-x_{i}=\mathrm{ST} \cos \theta_{1} .
$$

El valor de la subtangente ST debe ser el mismo en ambas, es decir:

$$
x_{i} / \cos \theta_{0}=\left(x_{1}-x_{i}\right) / \cos \theta_{1}
$$

por tanto 


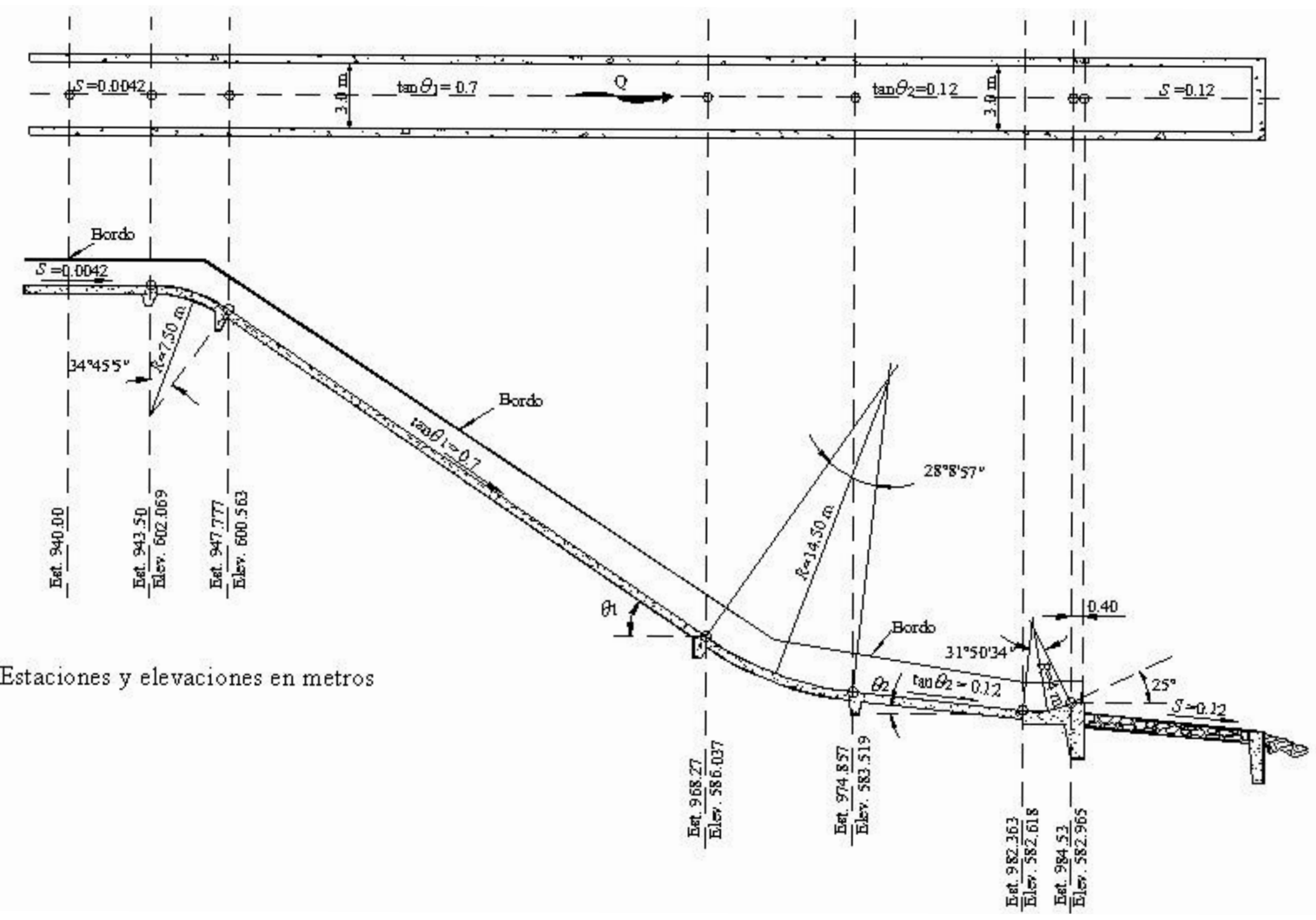

Figura 5. Canal del ejemplu 


$$
\begin{aligned}
x_{i}=\frac{x_{1} \cos \theta_{0}}{\cos \theta_{0}+\cos \theta_{1}} & =\frac{4.2768 \cos 024064^{\circ}}{\cos 0.24064^{\circ}+\cos 3499202^{\circ}} \\
x_{i} & =23509 \mathrm{~m}
\end{aligned}
$$

La subtangente resulta

$$
\mathrm{ST}=\frac{x_{i}}{\cos \theta_{0}}=\frac{2.3509}{\cos 0.24064^{\circ}}=2.3509 \mathrm{~m}
$$

Con ésta, el radio de la curva circular que pasa por los puntos 1 y 2 (tangente al fondo de ambos tramos del canal) vale:

$$
R=\frac{\mathrm{ST}}{\tan (\theta / 2)}=\frac{23509}{\tan (34.75138 / 2)}=75129 \mathrm{~m}
$$

valor que puede redondearse a $R=7.5 \mathrm{~m}$, que equivale a una curvatura constante $\kappa=-1 / 7.5=-0.13333 \mathrm{~m}^{-1}$; negativa por ser convexa y de valor constante en cualquier punto de la curva circular.

El centro del círculo tiene las coordenadas:

$$
\begin{gathered}
x_{0}=-R \operatorname{sen} \theta_{0}=-7.5\left(\operatorname{sen} 024064^{\circ}\right)=-0.0315 \mathrm{~m} \\
y_{0}=R \cos \theta_{0}=7.5\left(\cos 024064^{\circ}\right)=7.49998 \mathrm{~m}
\end{gathered}
$$

y con $R^{2}=(7.5)^{2}=56.25$, la ecuación de la curva es

$$
(x+0.0315)^{2}+(7.49998-y)^{2}=56.25
$$

o bien

$$
y=7.49998-\left[56.25-(x+0.0315)^{2}\right]^{1 / 2}
$$

de manera que

$$
\frac{d y}{d x}=\left[56.25-(x+0.0315)^{2}\right]^{-1 / 2}(x+0.0315)
$$

es la tangente del ángulo $\theta$ de inclinación de un recta tangente a la curva en cualquier punto de abscisa $x$.

Por otra parte, el cuadrado del primer término en la ecuación 10 b vale

$$
\frac{q^{2} \kappa^{3}}{g \cos \theta}=\frac{(5)^{2}(-0.133333)^{3}}{9.81 \cos \theta}=-\frac{0.0060407}{\cos \theta}
$$

para que se satisfaga dicha ecuación en la forma

$$
\frac{0.0060407}{\cos \theta}=\frac{\left(\lambda \kappa d_{c}\right)^{3}\left(1-\kappa d_{c}\right)}{\kappa d_{c}(1+\lambda)-1}
$$

donde $\lambda$ se calcula de la ecuación $4 ; \theta$ de las ecuaciones $a, b$ y c. Además, la energía específica mínima queda expresada por la ecuación 12. Si se efectúa un análisis numérico de la ecuación d, el segundo término crece con $d_{c}$, pero $\cos \theta$ se aleja del valor 1 para que dicha ecuación se satisfaga, lo que traslada a la sección crítica hacia aguas abajo del inicio de la curva. Sin embargo, esto hace que disminuya $E_{c}$ y con ésta el nivel de energía, lo que descarta la presencia de la sección crítica en puntos aguas abajo. Por tanto, dicha sección se debe presentar prácticamente al inicio de la curva, donde $\cos \theta=\cos \theta_{0} \cong 1$.

La solución de la ecuación d es con $\kappa d_{c}=-0176739$. En efecto, de la ecuación 4 se tiene

$$
\lambda=-\left(\frac{1+0.176739}{-0.176739}\right) \ln (1+0.176739)=1.08358
$$

Por tanto, se alcanza el valor

$$
\frac{\left(\lambda \kappa d_{c}\right)^{3}\left(1-\kappa d_{c}\right)}{\cdots \lambda 1,211}=
$$




$$
\frac{[1.08358(-0.176739)]^{3}(1.176739)}{(-0.176739)(2.08358)-1}=0.0060408
$$

que es prácticamente igual al que se debe satisfacer, siendo el tirante crítico

$$
d_{c}=-0.176739 /-0.133333=1.3255 \mathrm{~m}
$$

Con $\cos \theta \cong 1$ en la ecuación 12 , la energía específica crítica vale

$$
\begin{gathered}
E_{c}=1.3255\left[1+\frac{1}{2} \frac{1.08358(1+0.176739)}{1+0.176739(1+1.08358)}\right] \\
E_{c}=1.9431 \mathrm{~m}
\end{gathered}
$$

Ambos valores $\left(d_{c}\right.$ y $\left.E_{c}\right)$ son menores que los obtenidos para el fondo plano y por ello la sección crítica se presenta al inicio de la curva y en dicha sección se inicia el cálculo del perfil del flujo gradualmente variado que se forma antes y después del cambio de pendiente. La diferencia de las energías específicas críticas antes y dentro de la curva sirven para compensar las pérdidas por curvatura y fricción.

\section{Conclusiones}

En este trabajo se establecen las condiciones en que se realiza el flujo curvilíneo unidimensional en un canal de fondo curvo, de manera similar a la que sigue Dressler (1978) con el mismo grado de precisión. En este caso, la ecuación 10 muestra que el tirante crítico depende de las variables tradicionales en el flujo rectilíneo, además de la curvatura del fondo y del llamado coeficiente de curvatura. Según la ecuación 17, el parámetro $\mathrm{F}_{\mathrm{c}}$ no es un número fijo en ca na les de fondo curvo vertical, como ocurre con el valor 1 en flujo rectilíneo, y depende tanto del coeficiente de curvatura $\lambda$ como del propio tirante $d$. Sin embargo, el número de Froude $\mathrm{F}$ alcanza el valor 1 para el flujo crítico.

\section{Referencias}

Lenau W. y Cassidy J. (1969). Flow Through Spillway Flip Bucket. Journal of $\mathrm{H}$ ydrau lics Division, IAHR, Vol. 95, № HY2, marzo.

Dessler Robert F. (1978). New Nonlinear Shallow-Flow Equations With Curvature. Journal of H ydrau lics Research, IAHR, Vol. 16, № 3.

Prasad K. (1984). Pressure Distribution on Ski-Jump Buckets. Irri ga tion and Power, Nueva Delhi, Julio.

Sotelo G. (1998). Hidráulica general. Limusa, Vol 1. México.

Sotelo G. y Ruiz R. (1994). Flujo curvilíneo con vórtice libre. XIII Congreso Nacional de Hidráulica. Puebla, Puebla, México. 


\section{Semblanza de los autores}

G ilberto Sotel o-Á vila. Es ingeniero civil, maestro en ingeniería, doctor en ingeniería y Profesor Emérito de la UNAM. Es investigador nacional nivel 2 del Sistema Nacional de Investigadores del CONACYT y recibió en la UNAM el premio Universidad Nacional 1998, Docencia en Ciencias Exactas; el Consejo Técnico de la Facultad de Ingeniería le otorgó las cátedras especiales Javier Barros Sierra en 1999 y Mariano Hernández Barre nechea en 2000. El Colegio de Ingenieros Civiles de México le concedió los Premios Nacionales: Miguel A. Urquijo 1999, otorgado al mejor artículo técnico publicado durante 1997 y 1998, Mariano Hernández Barrenechea 2003 a la docencia y José A. Cuevas al mejor artículo técnico del 2002. Inició su actividad universitaria en 1958 como Investigador en el Instituto de Ingeniería y como profesor en licenciatura y posgrado dentro de la Facultad de Ingeniería, actividad que a la fecha todavía continúa. Fue jefe de la División de Ingeniería Civil, Topográfica y Geodésica, consejero técnico de la Facultad de Ingeniería y consejero académico de Área de la UNAM. Participó en la planeación, proyecto y supervisión de aproximadamente 150 obras de ingeniería civil dentro y fuera del país. Es vicepresidente del Comité Regional Latinoamericano de Hidráulica de la Asociación Internacional de Investigaciones e Ingeniería Hidráulica, miembro regular de la Academia Mexicana de Ciencias, del Colegio de Ingenieros Civiles de México, Miembro de Honor de la Academia de Ingeniería y del Consejo Académico del CENEVAL para el examen de calidad profesional de los ingenieros civiles. Es autor de dos libros de texto y coautor en tres más de consulta. Ha publicado 70 artículos de investigación y 11 de difusión, así como apuntes y notas de sus cursos.

I sis Ivette Valdez-Izaguirre. Ingeniera civil y candidata al grado de maestra en ingeniería civil. De 1996 a la fecha ha realizado actividad académica en el Laboratorio de Hidráulica de la Facultad de Ingeniería de la UN AM. Se ha desarrollado en el medio profesional de 1994 a 1996 en la planeación, proyecto y supervisión de 5 proyectos de ingeniería en Ingeniería Sanitaria y Saneamiento Inte gral S.A. de C.V. Es miembro regular y vocal de la XXVI Mesa Directiva de la Asociación Mexicana de Hidráulica; asimismo, es miembro regular del Colegio de Ingenieros Civiles de México. Es representante titular del área de hidráulica en la III Mesa Directiva del Colegio del Personal Académico de la Carrera de Ingeniero Civil de la Facultad de Ingeniería. De 1999 a la fecha ha colaborado en proyectos de investigación con el Dr. Gilberto Sotelo A. Actualmente interviene en la revisión y calibración de equipo e instalaciones del laboratorio de hidráulica y en la elaboración de material didáctico para la impartición de prácticas de laboratorio. del cual se han publicado las quías de prácticas de hidráulica 